\title{
Q Rugby headgear and concussion prevention: misconceptions could increase aggressive play
}

\author{
Richard Menger, MD, ${ }^{1,2}$ Austin Menger, ${ }^{3}$ and Anil Nanda, MD, MPH ${ }^{1}$ \\ ${ }^{1}$ Department of Neurosurgery, Louisiana State University of Health Sciences, Shreveport, Louisiana; ${ }^{2}$ Harvard University John F. \\ Kennedy School of Government, Cambridge, Massachusetts; and ${ }^{3}$ Georgetown University, Washington, DC
}

\begin{abstract}
OBJECTIVE Multiple studies have illustrated that rugby headgear offers no statistically significant protection against concussions. However, there remains concern that many players believe rugby headgear in fact does prevent concussions. Further investigation was undertaken to illustrate that misconceptions about concussion prevention and rugby headgear may lead to an increase in aggressive play.

METHODS Data were constructed by Internet survey solicitation among United States collegiate rugby players across 19 teams. Initial information given was related to club, age, experience, use of headgear, playing time, whether the rugger played football or wrestling in high school, and whether the player believed headgear prevented concussion. Data were then constructed as to whether wearing headgear would increase aggressive playing style secondary to a false sense of protection.
\end{abstract}

RESULTS A total of 122 players responded. All players were male. The average player was 19.5 years old and had 2.7 years of experience. Twenty-three of 122 players (18.9\%) wore protective headgear; $55.4 \%$ of players listed forward as their primary position. Overall, $45.8 \%$ (55/120) of players played $70-80$ minutes per game, $44.6 \%(54 / 121)$ played football or wrestled in high school, 38.1\% (45/118) believed headgear prevented concussions, and $42.2 \%(51 / 121)$ stated that if they were using headgear they would be more aggressive with their play in terms of running or tackling. Regression analysis illustrated that those who believed headgear prevented concussions were or would be more likely to engage in aggressive play $(p=0.001)$.

CONCLUSIONS Nearly $40 \%$ of collegiate rugby players surveyed believed headgear helped to prevent concussions despite no scientific evidence that it does. This misconception about rugby headgear could increase aggressive play. Those who believed headgear prevented concussion were, on average, 4 times more likely to play with increased aggressive form than those who believed headgear did not prevent concussions $(p=0.001)$. This can place all players at increased risk without providing additional protection. Further investigation is warranted to determine if headgear increases the actual measured incidence of concussion among rugby players in the United States.

http://thejns.org/doi/abs/10.3171/2016.1.FOCUS15615

KEY WORDS rugby; concussion; head injury

$\mathrm{R}$ UGBY is the third most popular sport in the world. England boasts more than 2.5 million registered players. ${ }^{12}$ Popularity in the United States is growing. USA Rugby has more than 450,000 registered players with 67,000 high school student athletes. The International Rugby Board economic data have noted that USA Rugby participation grew $350 \%$ from 2004 to 2011, and rugby is noted to be the fastest growing sport in the United States. ${ }^{8}$ However, injuries are part of the game; on average a team will have $18 \%$ of their roster unavailable due to injury for a given match. ${ }^{4}$ Across both academic and mainstream publications, medical interest in contact sports has shifted to concussion prevention and long-term neurocognitive decline.${ }^{29}$ Rugby is at the forefront of that discussion.

Concussion is a distinct and separate entity from that of mild traumatic brain injury. ${ }^{21}$ The current working definition states that concussion is a traumatically induced pathophysiological process affecting the brain. Concussion is distinguished by its rapid onset of impairment, spontaneous resolution of symptoms, and its overarching 
functional disturbance without structural abnormality. Nearly $80 \%-90 \%$ of concussion symptoms resolve in the first 7-10 days while children and adolescents may have a prolonged course of recovery. ${ }^{11,21}$ Focus has moved specifically toward prevention of secondary injury from a metabolically vulnerable postconcussive period.

It is estimated that approximately $1.6-3.8$ million sports-related brain injuries occur yearly in the United States. ${ }^{17}$ Concussion is the third most common injury in rugby; prospectively gathered data for rugby quantifies 91 injuries per 1000 playing hours. ${ }^{4}$ The incidence of injury is higher for organized rugby than professional hockey, soccer, or cricket. Four concussions per 1000 playing hours have been reported in the rugby "forwards" group, with an average of 14 days of participation missed. Nearly 5 concussions per 1000 playing hours have been reported among the rugby "backs" group with an average of 10 days of sport missed. ${ }^{4}$

Randomized prospective studies have shown that current and expanded headgear is statistically unreliable for protection against concussion. ${ }^{14,20,22}$ However, many players may incorrectly believe that rugby headgear in fact prevents concussions ${ }^{26}$ This study looks to explore the behavior and attitudes regarding the use of protective headgear among collegiate rugby players in the United States.

\section{Methods}

Data were constructed via internet survey solicitation among collegiate rugby players across 19 college clubs playing at the USA Rugby Division I-III level; men's rugby is currently not a National Collegiate Athletic Association (NCAA) regulated sport (http://usarugby.org/ncaa). Surveys were sent out to individual members of each club via contact information provided by team leaders. Participants answered questions providing information regarding current club, age, years of experience playing rugby, use of headgear, if having a concussion would incline them to use headgear, playing time, whether they played football or wrestled in high school, and whether they believed rugby headgear prevents concussions.

Protective headgear was defined as the soft cloth and foam scrum cap that is currently approved by the International Rugby Board. No hard material is allowed for player protection in the sport of rugby. Use of headgear was defined as at least 1 match (80-minute game) usage. This was done to intentionally incorporate as many players as possible that sought out any additional protection during at least one complete gameplay.

Current club information gave notice to the level of competitive rugby being played. Football and wrestling were used as markers for previous participation in the leading American secondary school contact sports. Questions were also asked about whether the participant would be more aggressive in tackling or running if he were using protective headgear. The survey is incorporated into Table 1.

ANOVA, univariate binary logistic regression, and multivariate binary logistic regression were used as appropriate. We first performed a preliminary univariate binary logistic regression analysis to identify significant predictors. Then, we ran the significant predictors in a

\section{TABLE 1. Internet survey solicitation of collegiate rugby players}

\begin{tabular}{l}
\hline Q1: What is your age? \\
Q2: What college club do you play rugby for? \\
Q3: How many years of rugby playing experience do you have? \\
\hline Q4: What position do you primarily play? \\
\hline 1) Forward \\
\hline 2) Back \\
Q5: Have you ever worn protective headgear (scrumcap) for at least \\
80 minutes (1 game)? \\
\hline 1) Yes \\
2) No \\
\hline Q6: How much playing time do you generally have during an 80 -min- \\
ute match? \\
\hline 1) 80 minutes \\
\hline 2) $60-70$ minutes \\
\hline 3) $50-60$ minutes \\
\hline 4) $40-50$ minutes \\
\hline 5) <40 minutes \\
\hline Q7: Did you play organized tackle football or wrestle in high school? \\
\hline 1) Yes \\
\hline 2) No \\
\hline Q8: Do you believe the protective headgear (scrumcap) prevents \\
concussions? \\
\hline 1) Yes \\
\hline 2) No \\
\hline Q9: If wearing protective headgear (scrumcap) would you change your \\
playing style to a more aggressive form of tackling and running? \\
\hline 1) Yes \\
2) No \\
Q10: Do you wear a scrumcap because you had a concussion/or \\
would you wear one if you had a concussion? \\
\hline 1) Yes \\
2) No \\
\hline
\end{tabular}

multivariate binary logistic regression to find the variables that were most significant in impacting increased aggressive play. All statistical analyses were performed using Minitab 17. All $p$ values were held to the conventional cutoff of alpha $=0.05$ when hypothesis testing for statistical significance of predictors. Point estimation and confidence intervals are both presented and used in analysis.

\section{Results}

Our survey produced 122 responses. All participants were male. The average age was 19.5 years old (range 18-24 years). The average player had 2.7 years of experience with $23.7 \%$ of players being 1st-year players. Of the 122 players surveyed, $23(18.9 \%)$ actually wore protective headgear during a rugby match. Nearly half of the players, $44.6 \%$ (54/121), were backs with $55.4 \%$ of players listing forward as their primary position; 55/120 (45.8\%) of players who responded played 70-80 minutes per game. Meanwhile, $21.7 \%$ (26/120) of players played 40 minutes or less. There was a strong representation of previous contact sport experience; $44.6 \%$ (54/121) played football or 
wrestled in high school. Complete descriptive statistics are found in Table 2.

Overall, 38.1\% (45/118) of USA Rugby collegiate players responding to our survey believed headgear (scrum cap) prevented concussions. Nearly one-third of players, $39 / 120(32.5 \%)$ would wear a scrum cap as a result of having a concussion. More importantly, 51/121 (42.1\%) stated that if they were wearing headgear they would be more aggressive with their play in terms of running or tackling. Correspondingly, 57.9\% (70/121) would not change their style of play. The results of initial preliminary univariate binary logistic regression of all variables are found in Table 3 . The univariate binary logistic regression noted use of headgear $(p=0.044)$ and belief in concussion prevention ( $p<0.001)$ as the only 2 factors statistically related to increased aggressive play.

Univariate binary logistic regression deemed age, years of experience, position, playing time, and previous contact-sport participation as not significantly related to increased aggressive play with the use of headgear (Table 3). Actual use of headgear during a rugby match showed a statistical trend to increased aggressive play on univariate analysis, but on the final multivariate analysis it was deemed not statistically significant $(\mathrm{p}=0.160)$ (Table 4). However, we are $95 \%$ confident that those players who believed headgear prevented concussions would be 2-9 times more likely to play with increased aggression while wearing headgear $(p=0.001)$. The average rugby player in our survey who believed headgear prevented concussions would be 4 times more likely to play with increased aggressive technique if wearing headgear.

TABLE 2. Descriptive statistics of 122 Division I-III USA Rugby collegiate players

\begin{tabular}{lc}
\hline \multicolumn{1}{c}{ Variable } & Response \\
\hline Mean age, years & 19.5 \\
\hline Mean no. of years of experience & 2.7 \\
\hline Position & \\
$\quad$ Forward & $55.4 \%(67 / 121)$ \\
Back & $44.6 \%(54 / 121)$ \\
\hline Use of headgear & \\
Yes & $18.9 \%(23 / 122)$ \\
No & $81.1 \%(99 / 122)$ \\
\hline Playing time, minutes & \\
$70-80$ & $45.8 \%(55 / 120)$ \\
$60-70$ & $11.7 \%(14 / 120)$ \\
$50-60$ & $12.5 \%(15 / 120)$ \\
$40-50$ & $8.3 \%(10 / 120)$ \\
$<40$ & $21.6 \%(26 / 120)$ \\
\hline Football/wrestling* & \\
Yes & $44.6 \%(54 / 121)$ \\
No & $55.4 \%(67 / 121)$ \\
\hline Belief that headgear prevents concussion & \\
Yes & $38.2 \%(45 / 118)$ \\
No & $62.8 \%(73 / 118)$ \\
\hline Increased aggressive play with use of headgear & \\
Yes & $42.2 \%(51 / 121)$ \\
No & $57.8 \%(70 / 121)$ \\
\hline
\end{tabular}

* Refers to participation in organized contact sport during high school.

\section{Discussion}

In our series, $38.1 \%$ of collegiate rugby players believed that headgear prevented concussions; $32.5 \%$ of the players surveyed stated they would begin to wear a scrum cap as a reaction to having a concussion. Most importantly, players who believed that headgear prevented concussions were more likely to increase their aggressive style of play ( $\mathrm{p}=$ $0.001)$.

Overall, our descriptive data compare with those reported in previous literature on the topic; $18.9 \%$ of players in our series wore protective headgear. This is on par with other studies within similar cohorts. In New Zealand, a country where rugby is popular, about $20 \%$ of players wear headgear. ${ }^{26}$ In Canada, this number was $27 \% .{ }^{26}$ Our study represented players with an average age of 19.5 years and 2.7 years of playing experience. This corresponds with other studies investigating rugby participation in North America. In Canada, a similar series represented players with 4.9 years of experience averaging 19.9 years old. ${ }^{26}$

\section{History of Rugby Headgear}

Traditionally, rugby is played without headgear or any additional padding. Standard kit includes shorts, long socks, jersey, and cleats without spikes. ${ }^{7}$ Other protective equipment is optional, including a mouthpiece. Historically, players would tape ears down to prevent laceration injury. ${ }^{19}$ Approved International Rugby Board headgear consists of soft cloth no thicker than $1 \mathrm{~cm}$ with a density of more than $45 \mathrm{~kg} / \mathrm{m}^{3} .{ }^{13}$ The modern day scrum cap was developed at Christ's College in East Finchley, London, by Eurig Evans with all of the team's starting 15 players donning the gear. ${ }^{9}$ Of note, fellow Christ College alumni C. J. Monro in 1869 is largely credited with bringing rugby to New Zealand, the reigning World Cup champion., ${ }^{1,25,32}$ Development was never historically geared toward concussion prevention.

\section{Rugby Headgear and Concussion Prevention}

Outside the United States, there has been much study regarding the possible role of headgear in preventing concussions. In 2004, Rugby World Cup video footage was reviewed to show a statistically significant decrease in superficial head injury by those wearing rugby headgear. ${ }^{14}$ This was most prominent among forwards, who tend to be more physical. However, no reduction in concussion was noted. Marshall et al. reviewed protective equipment through 304 club-level players in New Zealand. ${ }^{20}$ Headgear again led to a statistically significant reduction in both ear and superficial scalp lacerations without concussion protection. Both scrum caps and mouth guards illustrated no statistically significant reduction in concussions. ${ }^{20}$

McIntosh performed a randomized controlled trial of rugby players in Australia with modified headgear in the 13 - to 20 -year-old age groups over a 2 -year period. Comparison was made with the popular habit of not wearing headgear, standard available International Rugby Board headgear, and a new experimental headgear with added foam density. The intention-to-treat analysis showed no difference in the rates of head injury or concussion between controls and headgear arms. The final conclusion 
TABLE 3. Univariate binary logistic regression analysis comparing each variable to increased aggressive play while wearing headgear

\begin{tabular}{lccc}
\hline \multicolumn{1}{c}{ Variable } & p Value & OR & $95 \% \mathrm{Cl}$ \\
\hline Age & 0.221 & 0.8445 & $0.6416-1.1115$ \\
\hline Years of experience & 0.141 & 0.8768 & $0.7347-1.0465$ \\
\hline Position & 0.577 & 0.8125 & $0.3918-1.6848$ \\
\hline Use of headgear & 0.044 & 2.5646 & $1.0102-6.5108$ \\
\hline Belief in concussion prevention & $<0.001$ & 4.2824 & $1.9374-9.4656$ \\
\hline Playing time & 0.274 & 1.0723 & $0.1689-7.3799$ \\
\hline Football/wrestling & 0.133 & 1.7500 & $0.8415-3.6393$ \\
\hline
\end{tabular}

is that the board could not recommend modified headgear for reduction of concussions. ${ }^{22}$

This has also been tested biomechanically. Rugby collisions are noted to occur at impact velocities ranging from $7 \mathrm{~m} / \mathrm{sec}$ to $13.8 \mathrm{~m} / \mathrm{sec}^{16}$ Knouse et al. tested the impact attenuation of 2 different types of International Rugby Board-approved headgear with a repeated linear force of $2.4 \mathrm{~m} / \mathrm{sec}^{16}$ Initially headgear did show some attenuation of impact forces, but this dissipated over time. The occipital region had the greatest vulnerability due to the fact that most headgear devices close or tie in the posterior portion of the head. Interestingly, biomechanical differences did exist between the 2 types of foam padding configurations (solid and honeycomb).

These previous studies largely focused on international play in countries where rugby is a dominant organized competitive contact sport. Rugby in the United States is unique in that it blossoms from organized soccer, wrestling, and football. Indeed in our study nearly half of the athletes surveyed participated in wresting or football in high school.

Mirroring the United States, a survey of Canadian rugby players documented that $62 \%$ of players and $33 \%$ coaches believed headgear prevented concussions. ${ }^{26}$ The Canadian Rugby Union officially does not support the use of headgear for concussion prevention. ${ }^{26}$

Our data begin to shed light on a culture of misinformation among the development of relatively new rugby players in the United States. More than one-third of players responding to our survey believed rugby headgear prevented concussions. About one-third of players would wear a scrum cap as a result of having a concussion. However, any link between concussion prevention and rugby headgear is clearly not supported by the literature.

Interestingly, our data did not show a statistically significant relationship between previous high school contact sport participation (wrestling or football) and increased

TABLE 4. Multivariate binary logistic regression analysis comparing only the statistically significant variables from univariate analysis to increased aggressive play while wearing headgear

\begin{tabular}{lccc}
\hline \multicolumn{1}{c}{ Variable } & p Value & OR & $95 \% \mathrm{Cl}$ \\
\hline Belief in concussion prevention & 0.001 & 4.0128 & $1.7988-8.9516$ \\
\hline Use of headgear & 0.160 & 2.0496 & $0.7509-5.5947$ \\
\hline
\end{tabular}

aggressive play $(\mathrm{p}=0.133)$. This tends to suggest a more widespread culture of misinformation than the anticipated targeted cohort of individuals with previous contact sport experience. This is concerning because our data illustrated that players who held this false notion would play with a more reckless form. They are likely afforded a false sense of security. This can place all players at an increased risk. Concussion education and education on proper technique must be addressed when players begin to play at the college level. This is an entry point into the sport for many previous athletes. For those learning to play in youth or high school, education should begin there.

\section{Youth Rugby}

Concussion prevention will become even more important as rugby gains traction at the youth level in the United States. Kirkwood et al. performed a systematic review and illustrated a significant risk of concussion in children and adolescents playing rugby in the United Kingdom. ${ }^{15}$ In children and adolescents the incidence of concussion was $0.2-6.9$ concussions per 1000 playing hours. This created a probability of concussion ranging from $0.3 \%$ to $11.4 \%$. There are 40 cumulative playing hours per match, meaning there could be a concussion almost every third game. As such, there has been a call in the United Kingdom for standardization of protocol testing and implementation.

In the United States there has been tremendous response and traction for the prevention of youth concussions. In Louisiana, neurosurgeons served as point experts to develop state policies for youth athletics. In 2011 the Louisiana State Youth Concussion Act was passed, requiring information, evaluation, and coach education about concussion symptoms. ${ }^{18}$ Emphasis was placed on avoidance of repetitive injury and prevention of malignant edema in a vulnerable pediatric population. ${ }^{10}$

The Lystedt Law will have implications in all 50 states. The law requires medical clearance of youth athletes considered "at risk" for having suffered a concussion. ${ }^{6}$ Drafted in Washington state, it is the most comprehensive return-to-play criteria for athletes younger than 18 years. It rests on the benchmark principle of "when in doubt, sit them out." ${ }^{\text {"S }}$ School districts work with coaches to develop and implement educational platforms. Students and their families are also involved in the process, signing and reviewing information about concussions at the beginning of the season. After a player has been formally removed from play, only a licensed health care provider can pro- 
vide written clearance for participation. The law is named after Zachary Lystedt, a 13-year-old football player who suffered a severe injury after a concussion resulting in a bilateral craniectomy following a premature return to play in $2006 .^{6}$

\section{USA Rugby Concussion Guidelines}

USA Rugby has been very responsive to concussion safety. USA Rugby, following the lead of the International Rugby Board, has an official concussion policy regarding rugby participation. This includes the 5 R's of "Recognize, Remove, Refer, Recover, and Return." 30 Any player showing signs of concussion is removed from the match permanently and cannot return to play until cleared by a medical professional. Adults must be symptom free for at least 1 week and then follow a return-to-play 5-day graduated contact protocol. Players younger than 18 years must sit out a minimum of 2 weeks. They then begin the graduated return-to-play protocol. Specific step-by-step guides are available for coaches and athletes. ${ }^{30}$

\section{Role of the Neurosurgeon}

Given their unique training emphasis on traumatic brain injury and neurological critical care, neurosurgeons should continue to take ownership of these data to become active in the diagnosis, prevention, and management of this disease as well as the creation of protective guidelines for rugby. The National Football League (NFL) has taken leadership in prevention by appointing 2 neurosurgeons as co-chairs to develop concussion policy. This has translated into on-field evaluation and the development of off-field policy. With the assistance of neurosurgeon input, concussions were reduced by $50 \%$ on kickoffs by a decrease in the distance between players and the end-zone on kickoffs. ${ }^{2}$ Neurosurgeons, along with the NFL Head, Neck and Spine Committee, have developed the return-toplay guidelines for evaluation of at-risk players. ${ }^{24}$

These return-to-play guidelines spawned directly from the McCrory et al. 2009 Consensus Statement on Concussion and Sport; focus is a "safety first" protocol. ${ }^{21}$ No-go criteria include loss of consciousness, confusion, amnesia, new or persistent subjective findings (headache, dizziness), neurological physical examination findings (motor, sensory, cranial nerves) symptoms, and persistent or worsening examination indicative of a more serious neurological injury. Further sideline testing looks to grade the player's recall, orientation, counting, and overall awareness through a series of question rubrics. ${ }^{3}$ This creates an objective score that is compared with the athletes' baseline, guiding the return-to-play decision. It is performed by a trained health care professional, often times a neurosurgeon, at the professional level.

\section{Future Educational Considerations}

Future educational focus for rugby players should be on both concussion prevention and proper technique. Athletes have varying learning styles illustrating the needed for varying educational platforms. Education and subsequent knowledge can alter on-field decisions that athletes make regarding their concussion health. ${ }^{27}$ Errors in athlete's decisions largely stem from underappreciated injury severity related to signs and symptoms. It also flows from the natural apprehension of being withheld from competition.

A myriad of educational tools, including print, Internet, television, and video games, have been developed to help physicians, athletes, and coaches. The core concept rests on educating across multiple platforms to different audiences. Each target audience has specific needs. ${ }^{27}$

Physicians and neurosurgeons are the leaders and decision makers. In regard to concussion prevention and management, physicians benefit from interactive education sessions and reminder messages ${ }^{27}$ Continuing medical education can be extremely beneficial in this context. Passive dissemination of educational materials may educate physicians, but it does not necessarily translate to an eventual change in athlete's behavior. Provvidenza et al. recommended establishing concussion education sessions and seminars. ${ }^{27}$ Integrating concussion education into the training of physicians is also vitally important, both at the medical student and resident levels.

Coaches benefit best from a reflective process of learning. ${ }^{27}$ Specific inquiries made about how a coach would deal with specific scenarios at specific times work best. This includes reflection-in-action (during the action), reflection-on-action (within the action, but not during the activity), and retrospective reflection-on-action outside of the action present. It is important to realize that coaches are community leaders who are responsible for the athletic and personal development of young student athletes. It is also important to recognize that coaches also feel the demands of athletic competition. It is crucial that sports administration within the school and the conference seek to create an open forum for discourse and reflection.

Emphasis is on a much more engaging and interactive education for young athletes. Provvidenza et al. noted that didactic and printed materials are not effective in changing athlete's behavior. ${ }^{27}$ Players, young student-athletes, are by definition learners. As such, it is important that concussion education cross multiple boundaries of learning and intelligence. Platforms should be active, diverse, and tailored to the individual player whenever possible. There should be a focus on peer support. Again, the goal is to change the culture to maximize both player output and player safety. Peer-to-peer interaction is vital in this context.

Education for injury prevention in rugby has had some success. The RugbySmart program was developed to counter spinal injuries in rugby union play in New Zealand. ${ }^{28}$ The program focused on coaches and referees by providing education about physical conditioning, injury management, and safe techniques for rugby play. Spine injuries resulting in permanent disablement subsequently decreased. Eight permanent spinal injuries occurred from 2001-2005; the predicted number was 18.9. Most importantly, the number of permanent spinal cord injuries resulting from scrums (a technical formation involving intense physical contact) plummeted. One player was injured as opposed to the estimated 9. This was also seen in South Africa. A decrease in youth $(<19$ years old) injuries from tackles was seen when dedicated safety techniques were integrated into training. ${ }^{5}$

This illustrates the need for comprehensive education. 
Direct education about concussions is important. However, information and coaching about safe and proper technique can have a dramatic impact in injury reduction as well. New players must be taught the proper techniques of rugby scrums, rucks, tackling, and mauls.

\section{Limitations}

Limitations of our study are focused on its survey use. Specifically, this is a survey study of collegiate rugby players. This selection favors individuals who are educated and ranging in ages from 18-24 years. It does not address adolescent or youth development. It does not cover a greater cross-section of society. There are no professional considerations. No real-time observations regarding the actual play of the ruggers were available. Response rate to the questionnaire was approximately $30 \%$. Intuitively, the survey favors those rugby players who either directly or indirectly had experiences with concussion during their rugby career as they would be more inclined to respond to the survey. Lack of statistical association could indicate the test is not adequately powered to determine the relationship.

\section{Future Considerations}

Moving forward, neurosurgeons, not just neurologists and pediatricians, must continue to take an active role in the education of rugby players regarding the proper use of headgear in rugby participation. Neurosurgeons, given their critical care training background, are uniquely poised to make significant contributions to the prevention and treatment of on-field concussions.

Further study is warranted to determine if the use of headgear increases the actual incidence of concussions in rugby. In 1998, the International Rugby Board voiced concern that rugby headgear could increase the risk of concussions and injury by altering on-field behavior. ${ }^{23}$ This was echoed in 2003 when the International Rugby Board voiced concern that padded headgear could increase cervical injuries due to players performing tackles with incorrect form (leading with their head). ${ }^{16}$ In 2014, it was again noted that rugby headgear may increase concussions. ${ }^{23} \mathrm{~A}$ parallel was drawn to amateur boxing where concussion rates have plummeted below that of rugby after officials removed headgear from competition.

Our study focuses on behavioral responses from survey questioning and does not provide observational data. Future investigation is warranted to fully understand the impact of rugby headgear on the incidence of concussion.

\section{Conclusions}

Overall, $38.1 \%$ of collegiate rugby players in our survey believed rugby headgear prevented concussions. The consensus of literature on the topic does not support this view. This misconception can increase aggressive play. The average player who believed headgear prevented concussions was 4 times more likely to play with increased aggressive form than a player who believed headgear did not prevent concussions. This can place both these and other players at increased risk without providing additional protection. Further investigation is warranted to determine if head- gear increases the actual incidence of concussion among United States rugby players.

\section{Acknowledgments} study.

We thank the collegiate rugby players who participated in this

\section{References}

1. Beaslely W: Soldiers, surgeons, and a speaker. Surgeons' News. (http://www.surgeonsnews.com/spectrum/history/ monro-dynasty) [Accessed February 26, 2016]

2. Biggs B: Increase in touchbacks led to significant decrease in NFL concussions on kickoffs. Chicago Tribune. February 15, 2012. (http://articles.chicagotribune.com/2012-02-15/ sports/ct-spt-0216-bears-concussions-chicago-20120216_1_ concussion-care-elizabeth-pieroth-head-injuries) [Accessed February 17, 2016]

3. Briggs S: Headguards in rugby would add to the concussion problem, not solve it, says doctor. Telegraph. October 8, 2014. (http://www.telegraph.co.uk/sport/rugbyunion/ news/11150094/Headguards-in-rugby-would-add-to-theconcussion-problem-not-solve-it-says-doctor.html) [Accessed February 17, 2016]

4. Brooks JHM, Fuller CW, Kemp SPT, Reddin DB: Epidemiology of injuries in English professional rugby union: part 1 match injuries. Br J Sports Med 39:757-766, 2005

5. Burger N, Lambert MI, Viljoen W, Brown JC, Readhead C, Hendricks S: Tackle-related injury rates and nature of injuries in South African Youth Week tournament rugby union players (under-13 to under-18): an observational cohort study. BMJ Open 4:e005556, 2014

6. Centers for Disease Control \& Prevention: The Zack Lystadt Law: A Concussion Survivor's Return to Play. (http://www.cdc.gov/media/subtopic/matte/pdf/031210-Zackstory.pdf) [Accessed February 17, 2016]

7. Dietzen CJ, Topping BR: Rugby football. Phys Med Rehabil Clin N Am 10:159-175, 1999

8. Economist: Rugby in America: worth a try. February 3, 2011. (http://www.economist.com/node/18070557) [Accessed February 17, 2016]

9. The evolution of head protection. (http://youngrugger. weebly.com/blog/the-evolution-of-head-protection) [Accessed February 26, 2016]

10. Ferro S: NFL concussion expert Rich Ellenbogen: 'I really don't care who's to blame. We just have to move the science forward.' Popular Science. October 9, 2013. (http://www.popsci.com/article/science/nfl-concussionexpert-rich-ellenbogen-i-really-don\%E2\%80\%99t-carewho\%E2\%80\%99s-blame-we-just-have) [Accessed February 17, 2016]

11. Harmon KG, Drezner JA, Gammons M, Guskiewicz KM, Halstead M, Herring SA, et al: American Medical Society for Sports Medicine position statement: concussion in sport. Br J Sports Med 47:15-26, 2013

12. International Rugby Board: Rugby player numbers. (http://4.bp.blogspot.com/-yeSN53py7PI/TmXJ4UMiD1I/ AAAAAAAAAgs/54kHw_zo9AY/s1600/ IRB+Player+Numbers.jpg) [Accessed February 26, 2016]

13. iSport: Protective rugby gear. (http://rugby.isport.com/ rugby-guides/protective-rugby-gear) [Accessed February 17, 2016]

14. Jones SJ, Lyons RA, Evans R, Newcombe RG, Nash P, McCabe M, et al: Effectiveness of rugby headgear in preventing soft tissue injuries to the head: a case-control and video cohort study. Br J Sports Med 38:159-162, 2004

15. Kirkwood G, Parekh N, Ofori-Asenso R, Pollock AM: Concussion in youth rugby union and rugby league: a systematic review. Br J Sports Med 49:506-510, 2015 
16. Knouse CL, Gould TE, Caswell SV, Deivert RG: Efficacy of rugby headgear in attenuating repetitive linear impact forces. J Athl Train 38:330-335, 2003

17. Langlois JA, Rutland-Brown W, Wald MM: The epidemiology and impact of traumatic brain injury: a brief overview. J Head Trauma Rehabil 21:375-378, 2006

18. Louisiana Emergency Response Network: Louisiana Youth Concussion Act. (http://lern.la.gov/resources/concussionact/) [Accessed February 17, 2016]

19. Macdonald DJM, Calder N, Perrett G, McGuiness RG: Case presentation: a novel way of treating acute cauliflower ear in a professional rugby player. Br J Sports Med 39:e29-e29, 2005

20. Marshall SW, Loomis DP, Waller AE, Chalmers DJ, Bird YN, Quarrie KL, et al: Evaluation of protective equipment for prevention of injuries in rugby union. Int J Epidemiol 34:113-118, 2005

21. McCrory P, Meeuwisse W, Johnston K, Dvorak J, Aubry M, Molloy M, et al: Consensus Statement on Concussion in Sport: the 3rd International Conference on Concussion in Sport held in Zurich, November 2008. Br J Sports Med 43 (Suppl 1):i76-i90, 2009

22. McIntosh AS, McCrory P, Finch CF, Chalmers DJ, Best JP: Rugby headgear study. J Sci Med Sport 6:355-358, 2003

23. National Football League: NFL Head, Neck and Spine Committee's Protocols Regarding Diagnosis and Management of Concussion. (http://images.nflplayers. com/mediaResources/lyris/pdfs/NFL_Diagnosis_Mgmt_ Concussion.pdf)

24. National Football League: NFL Return To Play Protocol. (http://static.nfl.com/static/content/public/photo/2014/08/21/0 ap3000000381608.pdf) [Accessed February 17, 2016]

25. New Zealand Rugby: Rugby's history. (http://www.nzrugby. co.nz/about-us/history/rugby-s-history) [Accessed February 26, 2016]

26. Pettersen JA: Does rugby headgear prevent concussion? Attitudes of Canadian players and coaches. Br J Sports Med 36:19-22, 2002

27. Provvidenza CF, Johnston KM: Knowledge transfer principles as applied to sport concussion education. Br J Sports Med 43 (Suppl 1):i68-i75, 2009

28. Quarrie KL, Gianotti SM, Hopkins WG, Hume PA: Effect of nationwide injury prevention programme on serious spinal injuries in New Zealand rugby union: ecological study. BMJ 334:1150, 2007

29. Stoney E: Rugby takes a deep look at concussions. New York Times. August 23, 2012. (http://www.nytimes. com/2012/08/24/sports/rugby/24iht-rugby24.html) [Accessed February 17, 2016]

30. USA Rugby: Concussions: Knows the 5 R's. (http:// usarugby.org/concussions) [Accessed February 17, 2016]

31. Wash Rev Code 28A.600.190 (http://apps.leg.wa.gov/rcw/ default.aspx?cite=28A.600.190) [Accessed February 17, 2016]

32. Watters S: Charles Monro biography. New Zealand

History. (http://www.nzhistory.net.nz/people/charles-monro) [Accessed February 26, 2016]

\section{Disclosures}

The authors report no conflict of interest concerning the materials or methods used in this study or the findings specified in this paper.

\section{Author Contributions}

Conception and design: all authors. Acquisition of data: A Menger. Analysis and interpretation of data: R Menger, A Menger. Drafting the article: R Menger. Critically revising the article: R Menger. Reviewed submitted version of manuscript: $R$ Menger, Nanda. Approved the final version of the manuscript on behalf of all authors: R Menger. Statistical analysis: A Menger. Administrative/technical/material support: R Menger, Nanda. Study supervision: Nanda.

\section{Correspondence}

Richard Menger, Department of Neurosurgery, LSU Health Sciences Center-Shreveport, 1501 Kings Hwy., PO Box 33932, Shreveport, LA 71130-3932. email: richard.menger@gmail.com. 